\title{
Influence of the Moment in Mathematical Models for Open Systems
}

\author{
EVELINA PROZOROVA \\ Mathematical-Mechanical Department \\ St. Petersburg State University \\ Av. 28 , Peterhof, 198504 \\ RUSSIA
}

\begin{abstract}
Article is proposed, built taking into account the influence of the angular momentum (force) in mathematical models of open mechanics. The speeds of various processes at the time of writing the equations were relatively small compared to modern ones. Theories have generally been developed for closed systems. As a result, in continuum mechanics, the theory developed for potential flows was expanded on flows with significant gradients of physical parameters without taking into account the combined action of force and moment. The paper substantiates the vector definition of pressure and the no symmetry of the stress tensor based on consideration of potential flows and on the basis of kinetic theory. It is proved that for structureless particles the symmetry condition for the stress tensor is one of the possible conditions for closing the system of equations. The influence of the moment is also traced in the formation of fluctuations in a liquid and in a plasma in the study of Brownian motion, Landau damping, and in the formation of nanostructures. The nature of some effects in nanostructures is discussed. The action of the moment leads to three-dimensional effects even for initially flat structures. It is confirmed that the action of the moment of force is the main source of the collective effects observed in nature. Examples of solving problems of the theory of elasticity are given.
\end{abstract}

Keywords: angular momentum, conservation laws, no symmetrical stress tensor, Boltzmann equations, Langevin, Landau equations, open systems

Received: April 20, 2021. Revised: October 14, 2021. Accepted: November 2, 2021. Published: November $22,2021$.

\section{Introduction.}

Non-equilibrium processes form the bulk of naturally occurring processes. Mathematical models that reflect what is happening in nature with some certainty are a mathematical representation of reality. Study of no equilibrium processes presented in the form of integral or differential equations, is an essential part of scientific research. The differential laws of continuum mechanics are obtained in two ways: using the finite volume method [1] and using the Ostrogradsky Gauss theorem when replacing the integral over the surface with an integral over the volume, that is, taking the integral by parts with the further use of theorems on the conditions for the integral to vanish [2]. Iterm outside of the integral in the transition from the integral formulation to the differential formulation is not taken into account in mechanics. From the definition of pressure, both from the classical Boltzmann equation and the modified one, it does not follow that the hydrostatic pressure is one third of the sum of the pressures on the coordinate areas. It is believed that the equations of motion obtained from the Boltzmann equation correspond to the zero order for the Euler equations and the first order for the NavierStokes equations. Dividing the speed into divergent and rotor parts results in a speed different from the initial order. The situation is saved by the fact that this circumstance affects the viscous component of stresses, which is a first-order quantity. Hydrostatic pressure is a zero-order quantity. Thus using Pascal's law for equilibrium, the pressure is chosen equal to one third of the pressure on the coordinate areas. However, the theory remains 
the same when determining the different pressures on each of the sites, i.e. $p_{x}, p_{y}$, $p_{z}$. Description using one pressure value is possible under equilibrium conditions (Pascal's law), but for no equilibrium conditions the fact is not obvious. This is pointed out in the tutorial [2].

This is also indicated by the analysis of equations for potential flows. It should be noted that the orders of magnitude of the right and left sides of the Boltzmann equation do not coincide and it does not fulfill the law of conservation of momentum. The diffusion term, which is a first-order quantity, is not taken into account in the right-hand side. Taking into account the flows across the border of the elementary volume, as "Markov's" of the processes is preserved. At the same time, the "history" of the process is partially taken into account. An alternative to the Boltzmann equation is the Landau equation [3], It contains an additional (diffusion) term corresponding to the convective part. However, it does not take full account of particle collisions.

When solving the problem by the numerical method on modern machines, there is no need to discard the rotational component of the velocity. The classical mechanics of a continuous medium was based on the laws of the balance of forces, the law of conservation of moment was considered as a consequence of the fulfillment of the law of the balance of forces. We have proposed a model that takes into account the joint fulfillment of the laws and the corresponding equation of state, including the contribution of the rotational velocity component. When solving specific problems, the contribution of the distributed moment is often investigated, but the symmetry condition of the stress tensor is retained.

Sometimes, when solving problems of the theory of elasticity, the first invariants are used, but this can be done if the tensor is symmetric. It becomes symmetrical after discarding the rotational component. When considering vortex motion, the tensor is not symmetric (Love's method). The equilibrium condition is satisfied if there are no internal and external forces, however, any surface forces can be converted by changing variables into internal forces and deformations will occur. The role of the no symmetry of the stress tensor in the theory of elasticity is clearly shown in the work [4-6] in liquid and gas in [7-14]. It is necessary to emphasize that the stress tensor will be symmetric only under equilibrium conditions, but the results cannot be transferred to no equilibrium processes. The change in the angular momentum is associated with the appearance of an additional force, which can play the role of a small perturbation that affects the stability of the structure. The magnitude of the additional force is determined by the value of the gradient of physical quantities (density, speed, momentum) and the structure of the object under study. Numerical calculations and the experiment of work [15] can serve as evidence of the appearance of an additional force. The calculations were performed with a symmetric tensor, but without additional averaging over the unit cell. Due to the finite value of the step of the difference scheme, the effect of the presence of a moment was partially manifested. In the case of dynamic formation of the structure, the position of the center of inertia changes, which entails a change in the angular momentum. Writing out separately the law of equilibrium for forces and separately for moments of forces without taking into account their mutual influence, although the angular momentum creates an additional force, we come to the conclusion about the symmetry of the stress tensor. The effect of the moment on molecules is always manifested, even under equilibrium conditions, since there is a velocity distribution. Analyzing the results of solving the Euler equations and calculating potential flows, we obtain a vortex sheet [11], which indicates the existence of a moment. Mathematical analysis shows that the potential flow equations are not a subset of the Euler equations. An example of the influence of the vector nature of pressure is given, the influence of the stress tensor was presented earlier. Theoretical results have now been obtained mainly for plane problems with a symmetric stress tensor. However, in a complete with an non symmetric tensor, we have four unknowns and three equations. Therefore, the symmetry 
condition for the stress tensor is one of the conditions for closing the problem, but other conditions can also be chosen. In the work an examples of solving the problem of the theory of elasticity with a given distributed stress are given. An important contribution of the moment is observed for Coulomb plasma and in problems of the formation of fluctuations. Nanoparticles, even with a very low volumetric content (less than 1\%), are contained in such a fragment in a very large amount, and it is impossible to model their effect at this scale level. For example, a cubic fragment of a $1 \mu \mathrm{m}$ matrix contains more than thousand nanoparticles for a given volumetric content [16]. Numerical calculations of the effect of adding nanoparticles are based mainly on semiempirical theories. The use of the distribution function for an ensemble of Brownian particles (nanoparticles) is a promising direction of research [17], if the probabilistic term is replaced by a deterministic one, i.e. taking into account the action of the moment. The theory has been developed for other problems, but the technique can be used to calculate the effect of nanoparticles. The complex implementation and the need to use powerful computers limits the possibilities of its application. In the works carried out to simulate nanostructures and graphene, based on classical molecular dynamics, the choice of the potential plays an important role $[18,19]$. However, most of the works are devoted to experimental research. "The miniaturization of integrated circuits leads to the development and creation of nanoelectronics with a gigantic level of integration - hundreds of millions of transistors assembled on a single chip no more than a few square centimeters in size. The density of circuits in such integration is possible if the circuit elements have a size on the order of or less than $10 \mathrm{~nm}$. However, with such a density of elements, a so-called energy problem arises - the removal of dissipation energy from the circuit. If a solution to the problem is not found, then the generation of heat in the chip will lead to the impossibility of its proper functioning and a reduction in its service life." The paper also provides a review of methods for obtaining and studying nanoeffects [18]. The theoretical study of nanostructures due to the variety of methods for their preparation and variety of shapes is carried out both by continuous medium methods and statistical, mainly numerical. Dimensional effects are important here. The angular momentum plays a special role, creating spatial structures by virtue of its collective nature, violating their flat character. The emerging new effects are discussed.

\section{An angular momentum in mechanics.}

Model of a medium that takes into account rotational effects, leads to an non symmetric theory. The homogeneous Cosserat continuum in elastic theory, like other moment theories, assumes the existence of a material microstructure. However, the influence of the moment turns out to be important for a simple medium as well as for any collective motion of many particles. Consider the interaction of three particles interacting with each other. Since the moment is equal to the force acting on the center of inertia multiplied by the radius vector, we calculate the changes in the moment that occur after a certain period of time $r_{c}=\frac{m_{1} r_{1}+m_{2} r_{2}+m_{3} r_{3}}{m_{1}+m_{2}+m_{3}}$

at the next moment in time, the center of inertia will

$r_{c+\Delta c}=\frac{m_{1}\left(r_{1}+\dot{r_{1}} \Delta t\right)+m_{2}\left(r_{2}+\dot{r_{2}} \Delta t\right)+m_{3}\left(r_{3}+\dot{r_{3}} \Delta t\right)}{m_{1}+m_{2}+m_{3}}$

Consequently, an additional force arises, acting on each particle, since the gradient of the momentum's force is a force. Thus, the moment creates a collective force for any movement of particles with different speeds. The effect works in the formation of fluctuations and must be taken into account when calculating the equation of state. In continuum mechanics, integral conservation laws obtained experimentally are written in differential form. When passing to differential equations, the Ostrogradsky-Gauss theorem is used for the selected fixed volume, ignoring the possible rotation of the elementary volume. The discarded term represents the velocity circulation, its rotor part. Circulation is the action of the moment. Consequently, the rotational terms (moment) discarded in the 
construction of the Navier-Stokes equations are formed not only in the case of large gradients, but also due to the additional circulation of the velocity around the elementary volume. The mathematical definition of a point is different from the physical definition [3]. However, mathematics must simulate real environments. Therefore, an understanding of the losses caused by the action of approximations is necessary. The influence of the moment is manifested through pressure. There are no experiments confirming Pascal's law in nonequilibrium cases.

The kinetic theory (modified and classical) also does not follow Pascal's law. Consider the classical Boltzmann equation (the law of momentum does not hold)

$$
\begin{gathered}
\mathbf{u}(t, \mathbf{x})=\frac{1}{n} \int \xi f(t, \mathbf{x}, \xi) d \xi, \\
P_{i j}=m \int c_{j} c_{i} f(t, \mathbf{x}, \boldsymbol{\xi}) d \xi, \\
q_{;}=m \int c^{2} c_{i} f(t, \mathbf{x}, \boldsymbol{\xi}) d \xi \\
\boldsymbol{c}=\boldsymbol{\xi}-\mathbf{u} . \\
f\left(t+d t, \boldsymbol{r}+\boldsymbol{\xi}_{j} d t, \boldsymbol{\xi}_{j}+\boldsymbol{F}_{j} d t\right) d r d \xi_{j}= \\
f\left(\boldsymbol{r}, \boldsymbol{\xi}_{j}, \mathrm{t}\right) d \boldsymbol{r} d \xi_{j}+\left(\frac{\partial f}{\partial t}\right)_{c o l l} d t .
\end{gathered}
$$

$f$ is the distribution function, $\mathrm{r}$ is the radius vector; $x$ - point coordinate; $\xi$ is the velocity of a point, is the molecular weight, and, according to the definition of the distribution function $f_{N}$, the probability of finding the system at points $(\mathbf{x}, \xi)$ in the intervals $\boldsymbol{d} \boldsymbol{x}_{\boldsymbol{i}} \boldsymbol{d} \xi_{\boldsymbol{i}}$ is $\left(t, x_{1}, x_{2}, \ldots x_{N}, \xi_{1}, \xi_{2}, \ldots \xi_{N}\right) d x_{1} \cdot d x_{N} d \xi_{1} \cdot d \xi_{N}$. The proposed new equation has the form Taking into account rotation and diffusion, the equation () has the form

$f\left(t+d t, \boldsymbol{r}+\xi_{\boldsymbol{i}} d t+r \times \omega d t, \xi_{\boldsymbol{i}}+\boldsymbol{F}_{\boldsymbol{i}} d t+\right.$ $\left.\frac{\partial \mathrm{M}}{\partial \mathrm{r}} d t\right) d \boldsymbol{r} d \xi_{i}+G_{2}\left(t+d t, \boldsymbol{r}+\xi_{i} d t+r \times \omega d t, \xi_{i}+\right.$ $\left.\boldsymbol{F}_{\boldsymbol{i}} d t+\frac{\partial \mathrm{M}}{\partial \mathrm{r}} d t\right)=f\left(t, \boldsymbol{r}, \xi_{i}\right) \boldsymbol{d} \boldsymbol{r} \boldsymbol{d} \xi_{i}+G_{1}\left(t, \boldsymbol{r}, \xi_{i}\right)+$ $\left(\frac{\partial f}{\partial t}\right)_{\text {coll }} d t$.

$\mathrm{M}$ is the moment associated with the collective action of all particles on each other as a result of the displacement of the center of inertia, which is the result of the movement of particles with different speeds. $G_{1}$ and $G_{2}$ - flows through the boundaries of the considered elementary volume. These values are $G_{1}=m \xi_{i} \frac{\partial f}{\partial \mathbf{r}}$. They are responsible for the additional terms obtained by S.V. Vallander from phenomenological considerations [20]. The hydrodynamic description is constructed by averaging the corresponding physical quantities. The equations are obtained from the Boltzmann equation, multiplying it by the required value. It can be seen that in different directions for the same $\xi$ c -may differ. The same is indicated by the analysis of determining the pressure based on the potential flow, if the pressure is separately calculated using the potential function and the stream function [2123]. When obtaining a symmetric stress tensor, we have to use a symmetric equation of state. Therefore, the divergent part of the vector and the rotary part are distinguished. The latter is discarded. Under the selected additional requirements, the solution with a symmetric tensor turns out to be the only one. In the general case, there is no uniqueness if the pressure distribution is not specified. Consider the classical equations of aeromechanics

$\rho\left(\frac{\partial u}{\partial t}+u \frac{\partial u}{\partial x}+v \quad \frac{\partial u}{\partial y}+w \frac{\partial u}{\partial z}\right)=\rho f_{1}+\frac{\partial \sigma_{x x}}{\partial x}+\frac{\partial \sigma_{y x}}{\partial y}+$ $\frac{\partial \sigma_{z x}}{\partial z}$,

$\rho\left(\frac{\partial v}{\partial t}+u \frac{\partial v}{\partial x}+v \quad \frac{\partial v}{\partial y}+w \frac{\partial v}{\partial z}\right)=\rho f_{2}+\frac{\partial \sigma_{x y}}{\partial x}+\frac{\partial \sigma_{y y}}{\partial y}+$ $\frac{\partial \sigma_{z y}}{\partial z}$

$\rho\left(\frac{\partial w}{\partial t}+u \frac{\partial w}{\partial x}+v \frac{\partial w}{\partial y}+w \frac{\partial w}{\partial z}\right)=\rho f_{3}+\frac{\partial \sigma_{x z}}{\partial x}+\frac{\partial \sigma_{y z}}{\partial y}+$ $\frac{\partial \sigma_{z z}}{\partial z}$,

$y\left(\frac{\partial \sigma_{x z}}{\partial x}+\frac{\partial \sigma_{y z}}{\partial y}+\frac{\partial \sigma_{z z}}{\partial z}+\rho f_{3}\right)-z\left(\frac{\partial \sigma_{x y}}{\partial x}+\frac{\partial \sigma_{y y}}{\partial y}+\right.$ $\left.\frac{\partial \sigma_{z y}}{\partial z}+\rho f_{2}\right)+\sigma_{z y}-\sigma_{z y}=0$,

$x\left(\frac{\partial \sigma_{x y}}{\partial x}+\frac{\partial \sigma_{y y}}{\partial y}+\frac{\partial \sigma_{z y}}{\partial z}+\rho f_{2}\right)-y\left(\frac{\partial \sigma_{x x}}{\partial x}+\frac{\partial \sigma_{y x}}{\partial y}+\right.$ $\left.\frac{\partial \sigma_{z x}}{\partial z}+\rho f_{1}\right)+\sigma_{y x}-\sigma_{x y}=0$,

$x\left(\frac{\partial \sigma_{x z}}{\partial x}+\frac{\partial \sigma_{y z}}{\partial y}+\frac{\partial \sigma_{z z}}{\partial z}+\rho f_{1}\right)-z\left(\frac{\partial \sigma_{x x}}{\partial x}+\frac{\partial \sigma_{y x}}{\partial y}+\right.$ $\left.\frac{\partial \sigma_{z x}}{\partial z}+\rho f_{2}\right)+\sigma_{z x}-\sigma_{x z}=0$.

Usually we use Newton's equations, leaving the symmetric part of the operators. For example, $\frac{\partial u}{\partial y}=\frac{1}{2}\left(\frac{\partial u}{\partial y}+\frac{\partial v}{\partial x}\right)+\frac{1}{2}\left(\frac{\partial u}{\partial y}-\frac{\partial v}{\partial x}\right), \quad \tau=\mu \frac{\partial u .}{\partial n}$, $P=a \dot{S}+b E$.

Coefficients $a, b$ are scalars, $P$ is stress tensor (through $\sigma_{i j}$ ), $S$ is strain rate tensor, $\tau$ is friction.

We leave the first parenthesis. Returning to the equations (2), we find in this case six 
unknowns; to determine them, we have three equations of motion (taking into account the symmetry condition), the continuity equation, the energy conservation law and the equation of state. The solution is unambiguous. In the general case, we have nine tensor components plus three pressure components and to determine them there are six equations of motion, an equation of continuity, a law of conservation of energy, and there should be an equation of state for each direction. If we use the usual equation of state, then the equations are enough for us. We start solving a problem with a symmetric tensor and scalar pressure. Since pressure is a vector, the term created by the moment of pressure (additional twisting force) will appear in the equation of motion. From the laws of equilibrium of moments, we can determine the degree of no symmetry and perform an iterative procedure using the equations of motion. In fact, it will be necessary to solve ordinary differential equations for this particular case to determine the degree of no symmetry of the problem. The calculation

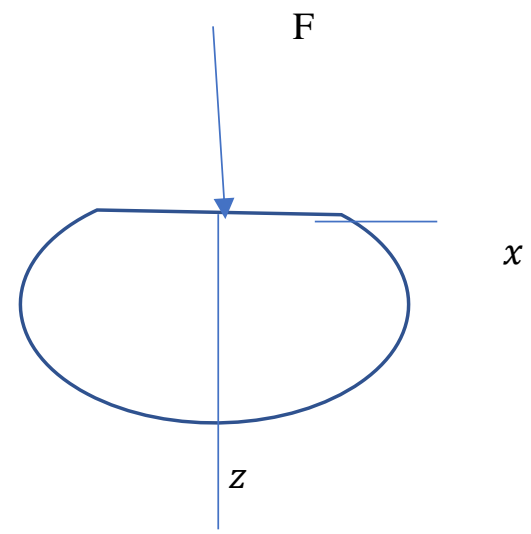

Fig. 1. Formulation of the problem

$$
\begin{gathered}
\sigma_{x}=-2 A z \int_{0}^{x} \frac{x d x}{\left(x^{2}+z^{2}\right)^{2}} \\
-A z \int_{0}^{y} \frac{d y}{\left(y+z^{2}\right)^{2}} \\
=A z \frac{1}{\left(z^{2}+y\right)}+f(z), \\
\sigma_{x}=A z \frac{1}{\left(z^{2}+x^{2}\right)}+f(z) .
\end{gathered}
$$

technique can be traced especially clearly in the problems of the theory of elasticity.

\section{Tasks of the theory of elasticity.}

Consider the problem when a concentrated force is given on a half-plane. Let us find the stress distribution with and without taking into account the influence of the moment (Fig. 1). General view of equations

$$
\begin{aligned}
& \frac{\partial \sigma_{x}}{\partial x}+\frac{\partial \sigma_{z x}}{\partial z}=0, \quad \frac{\partial \sigma_{z}}{\partial z}+\frac{\partial \sigma_{x z}}{\partial x}=0, \\
& x\left(\frac{\partial \sigma_{x z}}{\partial x}+\frac{\partial \sigma_{z z}}{\partial z}\right)-z\left(\frac{\partial \sigma_{x x}}{\partial x}+\frac{\partial \sigma_{z x}}{\partial z}\right)+\sigma_{z x}- \\
& \sigma_{x z}=0 .
\end{aligned}
$$

Suggested that $\sigma_{z x}=-A \frac{x}{x^{2}+z^{2}}$, option for case $\sigma_{x z}=\sigma_{z x}$ and $\sigma_{z}=-A \frac{z^{3}}{\left(x^{2}+z^{2}\right)^{2}}$ was considered in [4]. First, we solve the problem when the symmetry condition of the stress tensor is satisfied $\sigma_{x z}=\sigma_{z x}$.

$$
\frac{\partial \sigma_{z x}}{\partial z}=-A \frac{2 x z}{\left(x^{2}+z^{2}\right)^{2}},
$$

$$
\begin{gathered}
\frac{\partial \sigma_{x z}}{\partial x}=-A\left(\frac{1}{x^{2}+z^{2}}-\frac{2 x^{2}}{\left(x^{2}+z^{2}\right)^{2}}\right) \\
=-A\left(-\frac{z^{2}-x^{2}}{\left(x^{2}+z^{2}\right)^{2}}\right) . \\
\sigma_{z}=-A \int_{0}^{z}\left(\frac{1}{x^{2}+z^{2}}-\frac{2 x^{2}}{\left(x^{2}+z^{2}\right)^{2}}\right) d z= \\
-A \frac{1}{x} \operatorname{arctg} z-A\left(\frac{1}{x} \frac{z}{\left(1+(z)^{2}\right)}+\frac{1}{x} \operatorname{arctg} z\right)= \\
=-A \frac{1}{x} \frac{z}{\left(1+(z)^{2}\right)}+f(x) .
\end{gathered}
$$


Let $\sigma_{x}$ and $\sigma_{z x}$ remain the same. Then from the equation for the moments it is possible to determine the difference a new value $\sigma_{x Z}$ $\frac{\partial \sigma_{x z}}{\partial x}-\frac{1}{x} \sigma_{x z}+\frac{\partial \sigma_{z z}}{\partial z}=0$ $x\left(\frac{\partial \sigma_{x z}}{\partial x}+\frac{\partial \sigma_{z z}}{\partial z}\right)+\sigma_{z x}-\sigma_{x z}=0$.

Then a new value $\sigma_{z}\left(\sigma_{z}=\sigma_{z z}\right)$ can be determined. An example is given in order to demonstrate the many possible solutions in the two-dimensional case. Consequently, the solution of the two-dimensional problem under the condition of symmetry of the stress tensor is only one of the options for solving the problem. Algorithm for finding stresses with no symmetric tensor is easily to programme using previously obtained results. Task two. Stretching of a terminated plate under the action of a uniformly distributed load at the end. The problem was investigated in [4], we will check the methodology and propose a new solution. The solution to this problem is of interest to us as an opportunity to illustrate a method that has never been used before. Let us consider the tension of a plate with an embedment of infinite width with a thickness of $2 \mathrm{a}$ with a constant surface load of intensity $\sigma_{0}$ at the end. The stresses were represented.

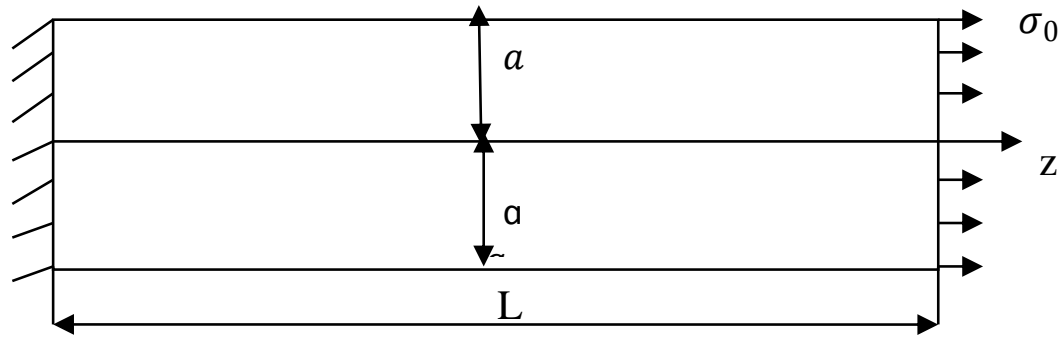

Fig.2. Stretching of a terminated plate under the action of a uniformly distributed load at the end

$$
\left\{\begin{array}{c}
\sigma_{z}=\sigma_{0}+c G e^{-m z}\left(\frac{x^{2}}{a^{2}}-\frac{1}{3}\right), \\
\tau_{x z}=c m G e^{-m z}\left(\frac{x^{3}}{3 a^{2}}-\frac{x}{3}\right), \\
\sigma_{x}=\frac{c}{v} G e^{-m z}\left(\frac{x^{2}}{a^{2}}-1\right), \\
\tau_{z x}=\frac{c}{v} G \frac{e^{-m z}}{m} \frac{2 x}{a^{2}} .
\end{array}\right.
$$

The solutions do not meet the symmetry condition for the stress tensor. Equation for moments

$$
\tau_{x z}-\tau_{z x}+\frac{\partial \mu_{z y}}{\partial z}=0
$$

The solutions do not meet the symmetry condition for the stress tensor.

Let the solution to the first equation be the first two lines of the parenthesis. Let's find a new solution. Let the tensor be symmetric. Then $\sigma_{x}=c m^{2} G e^{-m z}\left(\frac{x^{4}}{4 \cdot 3 a^{2}}-\frac{x^{2}}{3 \cdot 2}\right), \quad \mu_{z y}=0$.

For an no symmetric tensor, choose

$$
\mu_{z y}=-c G B e^{-m z} g(x) \text {. }
$$

Let's choose the old solution $\sigma_{z}, \tau_{x z}$. In this case $\tau_{z x}=c G A m e^{-m z}\left(\frac{x^{3}}{3 a^{2}}-\frac{x}{3}\right)$ for $g(x)=\left(\frac{x^{3}}{3 a^{2}}-\frac{x}{3}\right)$, $-c G A m^{2} e^{-m z}\left(\frac{x^{4}}{4 \cdot 3 a^{2}}-\frac{x^{2}}{3 \cdot 2}\right)$.

$\sigma_{x}=$

Therefore, the solution will be the fulfillment of the condition $1-A+B=0$.

Thus, we got a different solution to the problem.

\section{Some problems of statistical mechanics.}

In stochastic processes of open systems, the motion of fast molecules is associated with a change in the position of the center of inertia, which is accompanied by the appearance of a moment. The appearance of a moment leads to a change in the direction of velocities and the formation of local structures. It can be assumed that the moment will make a significant contribution to the equation of state for liquids and gases at high pressures (virial equation), since there are fast and slow molecules even for 
equilibrium distribution functions. The effect of the velocity distribution is most clearly manifested for locally equilibrium condit4ons in the Langevin, Fokker-Planck equations and in the Landau damping in plasma [24-30]. In the collision integral of the Boltzmann equation, the distribution function changes not only in space, but also in velocities. These changes are taken into account in the listed equations. Taking into account these or those changes is dictated by which "points" are investigated [3]. The phenomenon of Brownian motion is well known. At each moment in time, the motion of a Brownian particle is determined by the resultant forces from collisions with individual molecules of the environment. Due to the chaotic movement of molecules, this leads to a very complex and incessant movement of Brownian particles. The phenomenon of Brownian motion was the impetus for the creation of the theory of nonequilibrium fluctuations. Thanks to the work of Langevin, Einstein and Smoluchowski, the foundations of the modern theory of Brownian motion were laid. The paper proposes to replace the probabilistic forces in these equations by deterministic ones associated with the influence of the angular momentum. Possessing different speeds, as a result of their movement, the molecules change the position of the center of inertia of the system, which leads to the formation of an individual moment and an acting force on individual particles, which is the reason for the formation of diffusion processes. We emphasize that this process is not dissipative and is not directly related to collisions. As example we can investigate damping of longitudinal oscillations of an electron plasma (Landau damping), kinetic equations of Langevin and Fokker-Planck

Let us consider oscillations in a plasma without collisions, that is, let us proceed to the study of waves propagating in a plasma, the frequency of which is high in comparison with the frequency of pair collisions of electrons and ions. In this case, there are several options to consider. Landau collisional damping for large Knudsen numbers; for small Knudsen numbers in unbounded plasma; for small Knudsen numbers in a confined plasma. They differ from each other. When studying oscillations, we will consider small deviations from equilibrium [3,24-30].

Since we are interested in wave attenuation, we need to consider the plasma dielectric constant $\varepsilon$, which is determined by the attenuation coefficient $\gamma$. First, let's trace the waves in the "cold" isotropic plasma. The variant corresponds to the "collisionless" wave approximation. In this case, the Maxwell distribution functions

$$
\begin{aligned}
& f_{e}^{(0)}=\frac{1}{\left(2 \pi m_{e} k_{b} T\right)^{3 / 2}} \exp \left(-\frac{p^{2}}{2 m_{e} k_{b} T}\right), \quad f_{i}(p)= \\
& \delta(p) . \frac{r_{D}}{l} \ll \frac{\lambda}{l} \ll 1,
\end{aligned}
$$

the damping is determined by diffusion, but not by the Landau damping. The influence of the thermal motion of plasma particles on such oscillations is always small [3].

$\operatorname{Here} f_{e}^{(0)}$ is the equilibrium distribution function, $l$ is the mean free path $r_{D}$ is the Debye radius, $\lambda$ is the wavelength, the rest of the notation is generally accepted. Consider an unbounded plasma for small Knudsen numbers $l \ll \lambda$. Diffusion works here as well. Let us consider the dispersion and damping of longitudinal oscillations of an electron plasma under the influence of the thermal motion of plasma particles. Let us investigate a variant of a limited plasma, a free-molecular flow with a region of wavelengths (values of wave numbers) for which the contribution corresponding to Landau damping is the main one.

$l \rightarrow L(l \gg L), \quad r_{D} \ll \gamma \ll \sqrt{r_{D} L} \quad\left(r_{D} \ll L \ll\right.$ l).

We must use the Vlasov kinetic equations $[23,24]$ with a self-consistent field. Since we are interested in high-frequency oscillations, for which $\omega \tau \gg 1$, where $\tau$ is the average time between pair collisions of particles, we can ignore the integrals of particle collisions in the kinetic equations. Longitudinal oscillations of an electron plasma in the classical case are described by the following two equations (collisionless case, Vlasov equation)

$$
\frac{\partial \delta f}{\partial t}+v \frac{\partial \delta f}{\partial r}+e \delta E \frac{\partial f_{0}}{\partial p}=0,
$$


$\operatorname{div} \delta E=4 \pi \int d p \delta f$.

$$
\begin{aligned}
& \varepsilon_{l}(\omega, k) e \int d p \delta f(p, k, \omega) \\
& =i \int d r e^{-i k r} e \int d p \frac{\delta f\left(p, r, t_{0}\right)}{\omega-k v} .
\end{aligned}
$$

Suggested variant is

$$
\begin{aligned}
& \frac{\partial \delta f}{\partial t}+v \frac{\partial \delta f}{\partial r}+e \delta E \frac{\partial f_{0}}{\partial p}+\frac{\partial \delta M}{\partial r} \\
& +\frac{\partial}{\partial r} D \frac{\partial \delta f}{\partial r}=0 \\
& \operatorname{div} \delta E=4 \pi \int d p \delta f .
\end{aligned}
$$

$\varepsilon_{l}(\omega, k) e \int d p \delta f(p, k, \omega)=$

$i \int d r e^{-i k r} e \int d p \frac{\delta f\left(p, r, t_{0}\right)}{\omega-k v}+$

$i \int d r e^{-i k r} \int d p \frac{\delta M(p, r, t)}{\omega-k v} \frac{\partial f_{0}}{\partial p}$.

Qualitatively, we can say that for this case, diffusion plays a small role and, since part of the energy is converted into rotational motions (the action of the moment), the reversible operator will act as a dissipative one. Note that at the initial moment, the distributed moment of force also exists and concentrates a certain amount of energy. For monochromatic waves of large amplitude, the action can lead to the formation of a vertical velocity component, forming complex plane flows. Despite the collisionless nature of the movement binary collisions exist, as follows from work [31]. They create additional dissipation. It should be noted that the generalized equation for a unified description of kinetic and gas-dynamic processes is suitable for "weak" interactions. As before, the contribution of the angular momentums in the motion of molecules is not taken into account. Most likely, the difference between the most probable and average values is due precisely to the lack of taking into account the rotational movements for which the moment is responsible. Similar effects will be essential for Brownian motion. The theory of Brownian motion is one of the main branches of the statistical theory of open systems. Fluctuation (from Latin fluctuatio - fluctuation) - any random deviation of any value. In mechanics, a deviation from the mean value of a random variable characterizing a system of a large number of chaotically interacting particles. In the theory of Brownian motion elementary objects are small particles, while in kinetic theory, the main objects are molecules. Both models are macromodels, but the level of description of the structure of the environment is different. Fluctuations exist both in nonequilibrium states and in unsteady processes; in their absence, relaxation would be a "smooth" process and they could be described by single-valued functions of time. The presence of thermal fluctuations causes random deviations of real processes from such a "smooth" flow. The kinetic equation corresponds to a more detailed description. We believe that the environment is in equilibrium. We will consider two approaches to solving problems: the equation for a single particle and for an ensemble of particles (the Fokker-Planck equation) To take into account the atomic structure of a liquid, Langevin introduced an additional force into the equations of motion

$$
\begin{gathered}
F_{L}=m y(t), \quad F=-m \gamma v, \quad \gamma=\frac{6 \pi a}{m} \eta, \\
\eta=\rho v .
\end{gathered}
$$

Equations

$\frac{d r}{d t}=v, \frac{d p}{d t}+\gamma p=F_{0}+m y(t), F_{0}=$ $-\operatorname{grad} U . F_{0}-$ external force.

$$
<y_{i}(t)>,<y_{i}(t), y_{i}\left(t^{\prime}\right)>=2 D \delta_{i j}(t-
$$

$\left.t^{\prime}\right)$, the coefficient $D$ was determined by Einstein.

First, about a single particle. Let us repeat the reasoning performed in [3], but replace y (t) with the moment of force $M_{i}$ calculated for a given period of time. It can be calculated using the operation algorithm. As before, we assume that the characteristic correlation time of the values of the Langevin force is $\tau_{c o r}^{L} \ll \tau_{r e l}=$ $\frac{1}{r}$. As a result, we arrive at an expression for two time moments:

$$
\begin{gathered}
<M_{i}(t)>=0,<M_{i}(t) M_{j}\left(t^{\prime}\right)> \\
=2 D \delta_{i j}\left(t-t^{\prime}\right) . \\
D=\gamma \frac{k_{b} T}{m}-\text { - Einstein's coefficient }
\end{gathered}
$$

parenthesis means a function from a function (functional)

When using the kinetic description of Brownian motion, it is necessary to introduce an ensemble of noninteracting Brownian 
particles - the corresponding Gibbs ensemble. In this case, we represent the ensemble of Brownian particles as a continuous medium. However, the difference lies in the use of the "Hamiltonian" formalism for moving particles; for a continuous medium, in this case, the Langevin equation is used. Fokker-Planck equation in the kinetic theory has the form

$$
\frac{\partial f}{\partial t}+v \frac{\partial f}{\partial r}-\frac{1}{m} \frac{\partial U}{\partial r} \frac{\partial f}{\partial v}=D \frac{\partial^{2} f}{\partial v^{2}}+\frac{\partial}{\partial v}(\gamma v f) .
$$

The equation of A. Vlasova

$$
\begin{gathered}
\left\{\frac{\partial f}{\partial t}+v \frac{\partial f}{\partial r}+e\left(E+\frac{1}{c}[v B]\right) \frac{\partial}{\partial p}\right\} F(r, p, t) \\
=0 .
\end{gathered}
$$

Here $E, B$ are the total electric and magnetic fields, which are composed of external and self-consistent fields generated by plasma particles. They satisfy Maxwell's equations.

In the classical case, equilibrium is possible between Brownian particles and the medium; the particles can be distributed evenly [3]. However, such an assumption can be considered unlikely due to the distribution of particles over velocities and the formation of new moments for individual particles due to the motion of the center of inertia. The fact is that in this case the action of the moment creates a force that distributes the particles not only in terms of velocities, but also in coordinates. Modified Fokker-Planck equation has the form:

$$
\begin{aligned}
\frac{\partial f}{\partial t}+v \frac{\partial f}{\partial r}-\frac{1}{m} & \frac{\partial U}{\partial r} \frac{\partial f}{\partial v}+\frac{1}{m} \frac{\partial M}{\partial r} \frac{\partial f}{\partial v} \\
& =D \frac{\partial^{2} y}{\partial v^{2}}+\frac{\partial}{\partial v}(\gamma v f)
\end{aligned}
$$

Thus, for the kinetic theory for a gas, for the Landau damping and the motion of Brownian particles, the nonuniform distribution of particles in velocities and coordinates is supported by the angular momentum and creates fluctuations in physical quantities that must be taken into account.

\section{A little about nanostructures.}

For nanoparticles, it is customary to distinguish between two types of size effects. One intrinsic or internal, due to specific changes in the surface, bulk and chemical properties of the particle. The other is external, which is a sizedependent response to external action of forces, which is not associated with an internal effect. We will be interested in the processes that arise during the movement of nanoparticles at a low concentration of them in the collective interaction of particles.

The usual binary consideration of particle interactions does not provide a complete picture. The movement of particles changes the position of the center of inertia and, therefore, changes the moment for an individual particle even in a closed volume. This is especially important for charged particles, for example, for quantum dots. Nanoclusters and nanostructures are formed and used in different environments and they can differ significantly from each other. There are several mathematical methods for studying nanostructures: continuous medium methods, kinetic theory methods, molecular dynamics method, Byrd's method, and quantum mechanics methods. As a rule, we are talking about clusters of mesomechanics, quantum dots. Thermoelectric effects, thermal effects, and self-organization leading to the formation of fullerenes are considered. We will dwell on some of the possible consequences influence of the moment. The first thing that should be noted that the angular momentum is the collective action of many particles and acts almost always, since there is always a velocity distribution of molecules at temperatures above zero. This means that it is impossible to form stable flat structures without additional restraining forces. An example is the formation of a wavy surface of a gold film on silicon. If we consider quantum dots or fullerenes as Brownian particles, using the Langevin equation instead of Newton's equation, then the problem can be reduced to an equation. Vlasov. It can be assumed that from the point of view of the macrotheory, the process of entrainment of electrons by ballistic phonons, a review of the studies of which is given in [19], occurs as 
a result of the action of a moment on the entire molecule when a temperature gradient arises, which is a macroscopic effect. As the gradient decreases, the action of the moment force becomes insufficient for the joint motion of a heavy and a light particle, the latter being pulled forward. The effect of the moment on light electrons is more significant than on ions, and they are pulled forward. When approaching the boundary, gradients appear again and the role of the moment increases.

\section{Conclusion}

The paper proposes to take into account the influence of the angular momentum (force) in continued mechanics, kinetic equations, in stochastic processes and in some processes during the formation of nanostructures. The additional forces that arise are responsible for trancemute the initially flat nanostructure into three-dimensional. The main laws in physics and mechanics are the laws of conservation of mass, momentum, energy, angular momentum, charge, and some others. In the article it is shown that not all of the forces are enter for a complete description of the interacting particles and continued mechanics Any redistribution of particles is accompanied by the emergence of collective effects, which is associated with the action of the angular momentum and, consequently, with the action of an additional force. The effect always manifests itself, regardless of the branch of science: the formation of fluctuations, structures, quantum mechanics and some others. When constructing a theory, it is impossible to restrict oneself to potential forces that depend only on the distance between particles, since when the particles move, the center of inertia shifts, forming a moment. In continuum mechanics, for example, the stress tensor loses its symmetry for this reason. Some modification of the theory is suggested in open systems. Some of the problems of continuum mechanics cannot be solved using a symmetric stress tensor constructed by rejecting the influence of the moment (rotation of an elementary volume). The paper proposes a way to take into account the influence of the moment and, using specific examples, a calculation algorithm is proposed. In the mechanics of open systems for stochastic processes, it is proposed to replace the probabilistic terms with deterministic ones that determine the collective interaction of the moments of forces acting on individual particles.

\section{Reference}

[1] V.I. Smirnov. Higher mathematics course. T.2. M .: Nauka, 1974 .. 655.

[2] L.G. Loytsyansky. Mechanics of liquid and gas. M .: Nauka. 1970. 904.

[3] Y.L. Klimontovich. Statistical theory of open systems. T. 1.2. Moscow: Lenand, 2019

[4] E. A. Bulanov. Moment stresses in the mechanics of solid, bulk and liquid bodies. $\mathrm{M}$

.; University book. 2012.140.

[5]. A.M. Katz. The theory of elasticity. St. Petersburg. 2002.

[6] N.G .Kolbasnikov. Theory of metal pressure treatment. Resistance to deformation and ductility. St. Petersburg: St. Petersburg State University Publishing House, 2000. 314. [7]. E.V. Prozorova. Mathematical modeling of the processes of mechanics with large gradients. St.Petersburg. St. Petersburg University. 2005. 339.

[8]. E.V. Prozorova. The influence of dispersion in models of continuum mechanics. St.Petersburg. St. Petersburg University. 2013, 94.

[9]. Evelina Prozorova. Ostrogradsky-Gauss Theorem for Problems of Gas and Fluid. International Conference on Mathematical Models \& Computational Techniques in Science \& Engineering. 23-25 February 2019, London, UK. Mechanics. Journal of Physics: Conference Series Volume 1334. 2019. 012009 [10]. Evelina V. Prozorova. Influence of discrete model on derivatives in kinetic theory. JP Journal of Heat and Mass Transfer Volume 17, Number 1, 2019, Pages 1-19 ISSN: 0973-5763

[11]. E. V. Prozorova. Features of the rarefied gas description in terms of a distribution function. APhM2018. IOP Conf. Series: Journal of Physics: Conf. Series 1250 (2019) 012023. IOP Publishing. doi:10.1088/17426596/1250/1/012023 
[12]. Evelina Prozorova. The influence of the no symmetric stress tensor on the flow separation

Wseas Transaction on Applied and Theoretical Mechanics. DOI: 10.37394/232011.2020.15.9

[13]. Evelina Prozorova. The Effect of Angular Momentum and Ostrogradsky-Gauss Theorem in the Equations of Mechanic. Wseas transactions on flued mechanics DOI: 11.37394/232013.2020.15.2

[14]. Evelina Prozorova. Influence of the Angular Momentum in Problems Continuum Mechanics .Wseas transactions on applied and theoretical mechanics

DOI:10.37394/232011.2021.16.1

[15].Marek Barski, Adam Stawiarski, Pawe, J. Romanowicz, Bogdan Szybi?ski, Local Elastoplastic Buckling of Isotropic Plates with Cutouts under Tension Loading Conditions, International journal of mechanics, Volume 15, 2021, pp. 69-87.

[16]. M. V. Klychnikova, Kyaw Ye Ko, Investigation of Mechanical Properties of LowDensity Polyethylene with Copper Nanoparticles, International journal of mechanics, Volume 15, 2021, pp. 181-188.

[17]. Yu.A., Volkov, M.B. Markov Kinetic equations for a phonon gas. Keldysh Institute preprints. M.V. Keldysh. 2019. No. 83.15 p. doi: 10.20948 / prepr-2019-83 URL: http://library.keldysh.ru/preprint.asp?id=20198391, N.6, 2021.

[18] A. C. Dmitriev. Introduction to nanothermal physics. 2nd edition, electronic.

M .: Laboratory of knowledge. 2020

[19]. E. D. Eidelman. Thermoelectric effect and thermoelectric generator based on carbon nanostructures: achievements and prospects.
Advances in physical sciences. T. 191, N.6, 2021.

[20]. S.V. Vallander. Equations of motion of a viscous gas. Reports of the Academy of Sciences of the USSR, 1951, v13.

[21].S.M. Belotserkovsky, M.I. Nisht. Wing and aircraft aerodynamics. Aeromechanics.

M .: Science. 1976.9-24

[22]. V. V. Kozlov. Separation of the flow Novosibirsk State University.

https://vihrestruktura.3dn.ru/publ/2-1-0-6. [23]. S. V. Alekseenko, P. A. Kuibin, V.L. Okulov, Introduction to the theory of concentrated vortices. Moscow-Izhevsk. Institute for Computer Research. 2005.504 p.

[24]. R. Balescu. Equilibrium and nonequilibrium statistic mechanics. A WileyIntersciences Publication John Willey and Sons. New-yourk-London...1975.

[25]. N.G. Van Kampen. Stochastic processes in physics and chemistry. North-Holland. 1984. [26]. A.I. Akhiezer, I.A. Akhiezer, R.V.

Polovin, A.G. Sitenko, K.N. Stepanov. Plasma electrodynamics. M.: Science, 1974

[27]. P. Silin. Introduction to the kinetic theory of gases. M .: Science. 1971.

[28].S. Ishimaru. Basic principles of plasma physics. M .: Atomizdat. 1975.

[29]. A.A. Vlasov. Nonlocal statistical mechanics. M.: Science 1978.

[30].Peter M"orters and Yuval Peres. Brownian .Notes on Brownian motion and related phenomena Deb Shankar Ray* Department of Physical Chemistry, Indian Association for the Cultivation of Science, Jadavpur, Calcutta 700032,. (February 2, 2008)

ol. 78 (1), p. 25-27.

[31]. F. Goodman, G. Vachmann. Dynamics of gas scattering by a surface. M .: Mir, 1980. 423.

\section{Creative Commons Attribution License 4.0 (Attribution 4.0 International, CC BY 4.0)}

This article is published under the terms of the Creative Commons Attribution License 4.0

https://creativecommons.org/licenses/by/4.0/deed.en_US 